\title{
Uso de ecuaciones alometricas sobre ArcGis para el cálculo de biomasa: Aplicación para la medición de la biomasa del Pinus Halepensis Mill de la Comunidad Valenciana
}

\author{
Aparisi-Navarro, Silvia ${ }^{a}$ Lengua, Ismael ${ }^{a}$; $\quad$ Moncho-Santonja, María $^{\mathrm{a}}$ \\ y Peris-Fajarnés, Guillermo ${ }^{\text {a* }}$ \\ ${ }^{a}$ C.I.T.G. Universitat Politècnica de València, camino de Vera s/n, 46022 Valencia; E-mail address: \\ *gperis@upv.es
}

\section{Resumen}

La medición de la biomasa es clave para la planificación, gestión y estimación de los recursos biológicos de una determinada zona. Éstos se encuentran relacionados directamente con los aspectos medioambientales de la zona analizada. En la actualidad el proceso de estimación o medición de la biomasa se realiza a partir de los datos recogidos en campo, sumado a una serie de operaciones posteriores que consumen una gran cantidad de tiempo y recursos manuales pese al uso de herramientas como las hojas de cálculo. En este trabajo precisamente se integran las experiencias y procedimientos de cálculo de biomasa de autores como Ricardo RuizPeinado dentro del conocido software ArcGis.

Se ha desarrollado una herramienta en el entorno de este software para la automatización de la estimación de biomasa del Pinus halepensis Mill a partir de ecuaciones alométricas y datos del Inventario Forestal Nacional (IFN), ya que es una especie muy representativa de la Comunidad Valenciana, figurando como especie dominante en el 62 por ciento de la superficie arbolada de la misma.

Esta herramienta, permitirá un ahorro de tiempo considerable para la obtención del dato, y su adaptación para el cálculo de biomasa de otras especies. El resultado de ésta herramienta es una tabla base de datos, cuya unidad mínima es la parcela forestal (según el IFN) de la cual obtenemos los datos de: recuento de Pinos carrascos de la parcela, pies por hectárea, alturas máximas y medias, área basimétrica, indicé de Hart-Becking y biomasa en kg por hectárea y toneladas por hectárea.

Palabras clave: biomasa, Pinus halepensis Mill, automatización, alometría, IFN. 


\section{Introducción}

La biomasa es la cantidad de materia viva, y se encuentra directamente relacionada con el secuestro de carbono en el ámbito forestal, es por tanto de gran importancia cuantificar este parámetro para una mejor evaluación y control de la problemática medioambiental.

Uno de los grandes problemas a los que se enfrenta la sociedad de hoy en día es el cambio climático. Según los expertos del IPCC (Grupo intergubernamental de Expertos sobre el Cambio Climático) "para limitar los riesgos del cambio climático es necesario reducir de forma sustancial las emisiones de gases de efecto invernadero" uno de éstos gases es el dióxido de carbono, responsable al $50 \%$ del calentamiento global.

Los árboles mediante la fotosíntesis absorben dióxido de carbono y expulsan oxígeno a la atmósfera, por lo que en el proceso de crecimiento del árbol existe un acto de secuestro de este gas. Por lo tanto el conocimiento del dato de biomasa puede ser muy útil para estudios atmosféricos, de calidad o medioambientales entre otros.

Para calcular la biomasa total de un árbol así como de cada fracción del mismo hay una gran diversidad de modelos aunque todos ellos derivan de funciones lineales o logarítmicas (Parresol, 1999). Los modelos más utilizados hoy en día son los alométricos, con forma potencial, y se calculan a partir del diámetro medio y de la altura total del árbol.

Existe una base de datos gratuita de donde extraer estos datos (diámetros y altura) llamada Inventario Forestal Nacional. Hasta la fecha el dato de biomasa no es un dato directo consultable dentro de la base de datos del IFN, pero sí deducible a partir de los datos tomados en él, mediante ecuaciones alométricas.

Aplicando las ecuaciones alométricas desarrolladas por Ruiz.Peinado et al (2011) se ha calculado la biomasa por fracción arbórea (fuste, ramas gruesas, ramas medias, ramas finas y raíces) para cada uno de los individuos de una parcela.

Esta tarea podría realizarse mediante hojas de cálculo o de forma manual, no obstante la organización de la base de datos del Inventario forestal nacional, reparte la información en diversas tablas y se debe ir buscando los datos necesarios en cada una de ellas, lo cual, resulta un trabajo agotador e incluso molesto.

Por ello y aprovechando las nuevas funcionalidades de los SIG de escritorio, se ha elaborado una herramienta en entorno ArcGis para la automatización de todo este proceso y agilizar así la obtención del dato y su georreferenciación, que tanta importancia tiene para la gestión forestal y por ende para el medioambiente. 


\section{Metodología}

\subsection{Estructura del Inventario Forestal Nacional}

El Inventario Forestal Nacional es un proyecto llevado a cabo por el Ministerio de Agricultura Pesca y Alimentación, para la obtención de la máxima información sobre los montes españoles. Se trata de un inventario continuo de todo el territorio español y se realiza cada 10 años. La unidad de medida es la parcela forestal, la cual está georrefernciada para poder comparar en inventarios posteriores la evolución de la misma.

La información tomada campo se estructura en una base de datos, que después es manipulada en gabinete para la obtención de otros parámetros derivados de los originales y su integración en un Sistema de Información geográfica. Existen pues dos bases de datos diferentes, la de campo y la de datos SIG.

Estas bases de datos son de acceso gratuito y se dividen por provincias. Cada una de estas bases de datos consta de las siguientes tablas:

Tabla 1. Tablas de la base de datos SIG del Inventario Forestal Nacional

\begin{tabular}{ll}
\hline \multicolumn{1}{c}{ Nombre tabla } & \\
\hline CambioEspecie & Grupo de especies para elaborar las tablas de existencias \\
CambioEspecieReg & Agrupación de las especies para los gráficos de generación \\
\hline EspDominante & Códigos para identificar la especie dominante en la tabla de Estratos \\
Estratos_exs & Existencias por Estratos. Datos dendrométricos procesados por estrato \\
Mayores_exs & Datos d existencias procesadas por pie \\
\hline Parcelas_exs & Datos de existencias procesadas por parcela \\
ParcPoly & Parcelas de la provincia, tesela y estrato al que pertenecen y fisiología de las mismas \\
Poligon & Teselas de la provincia, estrato al que pertenecen, superficie niveles de clasificación del \\
& suelo, especies principales con su ocupación y Fcc \\
\hline Tarifas IFN3 & Ecuaciones utilizadas en el proceso de datos \\
\hline
\end{tabular}

Fuente: Elaboración Propia 
Uso de ecuaciones alometricas sobre ArcGis para el cálculo de biomasa: Aplicación para la medición de la biomasa del Pinus Halepensis Mill de la Comunidad Valenciana

Tabla 2: Tablas de la base de datos de campo del Inventario Forestal Nacional

\begin{tabular}{|c|c|}
\hline Nombre tabla & Descripción \\
\hline Listado definitivo & Listado definitivo de parcelas de campo a apear en el IFN3. \\
\hline PCDatosMap & Tabla referente a los datos de las parcelas de campo. \\
\hline PCDetTabla & Codificación de diversos campos del estadillo, leyenda de PCParcelas \\
\hline PCEspMapa & $\begin{array}{l}\text { Tabla que detalla la posición especie, ocupación y estado de masa de las } 3 \text { especies } \\
\text { arbóreas principales presentes en la tesela a la que pertenece la parcela según el MFE }\end{array}$ \\
\hline PCEspParc & $\begin{array}{l}\text { Tabla en la que se resumen los datos de ocupación, estado de masa, origen y tipo de } \\
\text { tratamiento de las tres especies principales presentes en las parcelas de campo. }\end{array}$ \\
\hline PCMatorral & $\begin{array}{l}\text { Principales especies de matorral encontrado en las parcelas de campo, con su fracción de } \\
\text { cabida cubierta y altura media... }\end{array}$ \\
\hline PCMayores & $\begin{array}{l}\text { Características dendrométricas de los pies mayores medidos en las parcelas de campo en } \\
\text { el IFN3 }\end{array}$ \\
\hline PCMayores2 & $\begin{array}{l}\text { Características dendrométricas de los pies mayores medidos en las parcelas de campo en } \\
\text { el IFN2. }\end{array}$ \\
\hline PCNueEsp & $\begin{array}{l}\text { Listado de especies presentes por parcela según lo observado por el capataz responsable } \\
\text { del apeo de la misma. }\end{array}$ \\
\hline PCParcelas & $\begin{array}{l}\text { Resumen de la información tomada en cada parcela de campo (usos de suelo, } \\
\text { tratamientos del vuelo y del suelo, tipo de suelo...) }\end{array}$ \\
\hline PCRegenera & $\begin{array}{l}\text { Pies de regeneración por categoría de desarrollo, } n^{\circ} \text { y altura media de los de categoría } 4 \text { y } \\
\text { densidad de los de categoría inferior a } 4 \text {. }\end{array}$ \\
\hline PCTabla Esp & $\begin{array}{l}\text { Parámetros límite por especie y para esta provincia, aportados por la Dirección General } \\
\text { para la Biodiversidad. }\end{array}$ \\
\hline Uso2Nivel1 & Equivalencias entre los códigos de uso de suelo utilizados en el IFN2 y el IFN3 \\
\hline
\end{tabular}

Fuente: Elaboración propia

\subsection{Proceso de cálculo}

Para el cálculo de biomasa se han utilizado las ecuaciones alometricas de Ruiz-Peinado et al (2011)). Para los valores de altura total y diámetro medio del Pinus halepensis (extraíble de la tabla Mayores_Exs de los individuos con código 24, pino carrasco), separa la biomasa en fracciones para el fuste con corteza $\left(\mathrm{W}_{\mathrm{s}}\right)($ ec.1), las ramas gruesas de más de 7 $\mathrm{cm}$ de diámetro $\left(\mathrm{W}_{\mathrm{b} 7}\right)$ (ec.2), dependiendo de su importancia según los pies sean de más de $27,5 \mathrm{~cm}$ de diámetro o de menos, las ramas medias entre 2 y $7 \mathrm{~cm}\left(\mathrm{~W}_{\mathrm{b} 2-7}\right)(\mathrm{ec} .3)$ y las ramas 
finas de menos de $2 \mathrm{~cm}$ junto con las acículas $\left(\mathrm{W}_{\mathrm{b} 2+\mathrm{n}}\right)(\mathrm{ec} .4)$, e incluyendo la biomasa de las raíces $\left(\mathrm{W}_{\mathrm{r}}\right)(\mathrm{ec} .5)($ tabla 3$)$.

Tabla 3. Ecuaciones alométricas empleadas

\begin{tabular}{|c|c|c|}
\hline \multicolumn{3}{|c|}{ Ecuaciones alométricas } \\
\hline Fuste & $\mathrm{W}_{\mathrm{s}}=0,0139 * \mathrm{~d}^{2} * \mathrm{~h}$ & (ec. 1) \\
\hline Ramas gruesas & Si d $\leq 27,5 \mathrm{~cm}: Z=0 ;$ si $d>27,5 \mathrm{~cm}: Z=1$ & (ec. 2) \\
\hline & $\mathrm{W}_{\mathrm{b} 7}=[3,926 *(\mathrm{~d}-27,5)] * \mathrm{Z}$ & (ec. 3) \\
\hline Ramas medias & $\mathrm{W}_{\mathrm{b} 2-7}=4,257+0.00506 * \mathrm{~d}^{2} * \mathrm{~h}-0,0722 * \mathrm{~d} * \mathrm{~h}$ & (ec. 4) \\
\hline $\begin{array}{l}\text { Ramas finas } y \\
\text { acículas }\end{array}$ & $\mathrm{W}_{\mathrm{b} 2+\mathrm{n}}=6,197+0.00932 * \mathrm{~d}^{2} * \mathrm{~h}-0,0686 * \mathrm{~d} * \mathrm{~h}$ & (ec. 5) \\
\hline Raíces & $\mathrm{W}_{\mathrm{r}}=0,0785 * \mathrm{~d}^{2}$ & (ec. 6) \\
\hline TOTAL ARBOL & $\mathrm{W}_{\mathrm{s}}+\mathrm{W}_{\mathrm{b} 7}+\mathrm{W}_{\mathrm{b} 2-7}+\mathrm{W}_{\mathrm{b} 2+\mathrm{n}}+\mathrm{W}_{\mathrm{r}}$ & (ec. 7) \\
\hline
\end{tabular}

Fuente: Ruiz-Peinado et al. (2011)

Aplicando estas ecuaciones a cada individuo de la parcela obtenemos la biomasa de cada árbol. La suma de la biomasa de todos los arboles de la parcela será la biomasa total de la parcela, que después se extrapola a la cantidad de biomasa por hectárea y se almacena en una tabla intermedia para la posterior generación de la tabla resultado.

Para georreferenciar este dato, es necesario la utilización de una de las tablas de la base de datos de campo del Inventario Forestal Nacional (PCDatosMap) donde se pueden encontrar las coordenadas UTM y el huso de las mismas.

Por tanto, es necesario la generación de otra tabla intermedia con el código de estadillo (identificador de la parcela forestal) y coordenadas, operación muy sencilla a partir de la funcionalidad Table to Table de ArcGis que nos permitirá relacionar la biomasa de una parcela con su posición espacial.

La implementación de éstas ecuaciones se ha realizado en la aplicación Model Builder en entorno ArcGis.

Además de las ecuaciones de la tabla 3, se han implementado también los siguientes cálculos para cada parcela:

- Área basimétrica (relación entre la sección normal de un árbol y la superficie de terreno que ocupa)

$$
G=\pi *\left(\frac{\frac{\text { Dmedio }}{2}}{100}\right)^{2}
$$


Uso de ecuaciones alometricas sobre ArcGis para el cálculo de biomasa: Aplicación para la medición de la biomasa del Pinus Halepensis Mill de la Comunidad Valenciana

- $\quad$ Densidad de pies (por hectárea)

$$
\text { Densidad }=\frac{10000 * N_{\text {pies }}}{\text { Superficie }}
$$

- Índice Hart-Becking (relación entre el espaciamiento medio del arbolado y su altura dominante ${ }^{3}$ )

$$
H B=\sqrt{\left(\frac{10000}{\frac{\text { Densidad }}{H_{\text {max }}}}\right) * 100} \quad \text { (ec.10) }
$$

- $\quad$ Alturas media y maxima

\subsection{Resultados}

El resultado es una aplicación eficaz y veloz para el cálculo de biomasa. Esta herramienta minimiza el tiempo de cálculo, de hacerlo de mediante hojas de cálculo (4 horas para el caso de 32 parcelas a 18 segundos, llegando incluso a poder calcular la biomasa de 1100 parcelas en 43 segundos).

La aplicación genera una tabla de resultados que consta de los siguientes campos:

Tabla 4. Campos calculados con la herramienta

\begin{tabular}{ll}
\hline Campo: & Descripción \\
\hline Estadillo & Identificador de la parcela forestal \\
\hline X,Y & Coordenadas UTM en huso 30 de la parcela forestal \\
\hline Pies por hectárea & Radio y superficie de cada una de las parcelas \\
\hline Frecuencia & Número de individuos por hectárea. \\
\hline SUM_TotalArbol & Número de individuos por parcela \\
\hline MAX_Ht y MEAN_Ht & Suma de la biomasa de todos los árboles de la parcela \\
SUM_G & Altura máxima y Altura media \\
\hline MEAN_Dmedio & Área basimétrica \\
y MAX_Dmedio & Media de los diámetros medios y Máximo de los diámetros medios \\
\hline G_m2_ha & Área basimétrica en mº por hectárea \\
\hline W_kg_ha y W_t_ha & Índice Hart-Becking \\
\hline H_B & \\
\hline
\end{tabular}

Fuente: Elaboración propia 
La herramienta realizada (Figura 1) podrá ser modificada para el cálculo de biomasa de otras especies simplemente con el cambio de ecuaciones alométricas para especies como el abeto común, el pinsapo, sabina albar, pino canario, pino negro, pino piñonero, pino rodeno, pino silvestre o pinus uncinata.

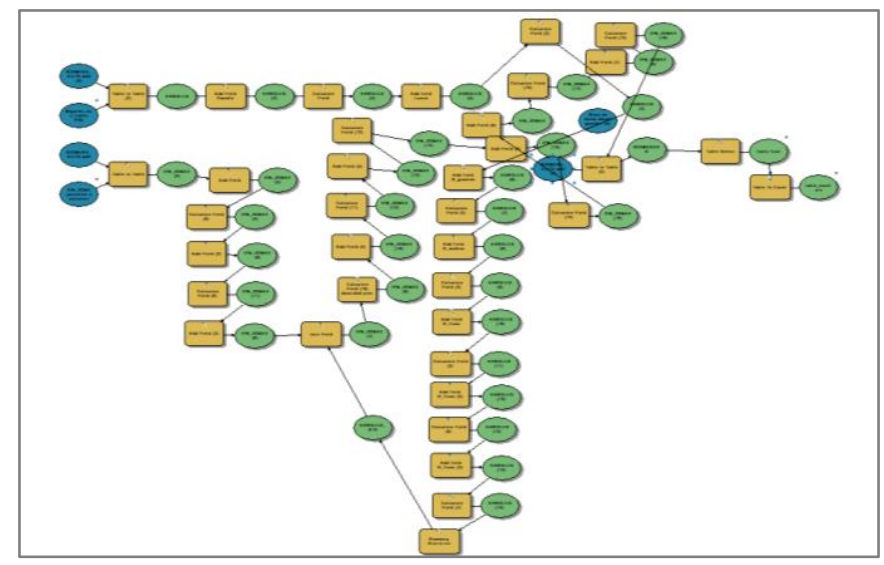

Figura 1. Flujo de trabajo de la herramienta. Fuente. Elaboración propia

Además al incorporar el resultado las coordenadas UTM es posible su conversión a capa cartográfica (capa de puntos) para su utilización en análisis forestales, entre otros.

Otra aplicación del dato de biomasa sería la obtención del carbono presente en la misma: aplicando el factor de $0,5 \mathrm{~kg}$ de carbono por $\mathrm{kg}$ de biomasa $(0,5 \mathrm{~kg} \mathrm{C} / \mathrm{kg})$ para la parte aérea y de $0,481(0,481 \mathrm{~kg} \mathrm{C} / \mathrm{kg})$ para la subterránea (Ritson y Sochacki 2003).

Estos porcentajes son para el pino piñonero pero el contenido de carbón en la biomasa se encuentra la mayoría de las veces entre el 45 y el 50\% de biomasa en materia seca. En muchas aplicaciones el contenido de carbón en la vegetación se estima a partir de una simple fracción de la biomasa, multiplicándola por 0.5 (Schlesinger, W .H. 1991).

El carbono presente a su vez se puede utilizar para la estimación del dióxido de carbono retenido utilizando la relación estequiométrica entre el carbono y el dióxido de carbono: $3.67 \mathrm{~kg} \mathrm{CO} 2 / \mathrm{kg}$.

\section{Conclusiones}

Esta herramienta supone un ahorro de tiempo en el cálculo de la biomasa para la especie Pinus halepensis Mill a partir del Inventario Forestal Nacional. 
Uso de ecuaciones alometricas sobre ArcGis para el cálculo de biomasa: Aplicación para la medición de la biomasa del Pinus Halepensis Mill de la Comunidad Valenciana

Permite a su vez el cálculo de otras variables importantes para la gestión forestal como el índice de Hart-becking o el área basimétrica.

Como sub-producto de esta herramienta se obtienen datos interesantes, como la biomasa por fracción de árbol (ramas finas, ramas gruesas, etc...) recuento de individuos o alturas máximas.

El dato obtenido se puede utilizar para la estimación del $\mathrm{CO}_{2}$ retenido en función de la biomasa en la zona donde se aplique la herramienta para un mejor estudio de problemas de la contaminación entre otros.

Por último, esta herramienta puede ser adaptada para otras especies, y permite la utilización del producto final en un Sistema de Información Geográfica, ya que puede ser transformado en capa cartográfica, para posteriores análisis SIG.

El proyecto podría ser ampliado y automatizado mediante el reconocimiento de áreas combinado con el reconocimiento de múltiples especies permitiendo la catalogación automática de cualquier área de manera interactiva. Esta capacidad permitiría a su vez facilitar los estudios de impacto relacionados con las modificaciones del territorio generadas por cualquier proyecto de obra civil, por cualquier alteración climática o por cualquier catástrofe medioambiental.

\section{Referencias}

Aparisi Navarro, Silvia, Coll Aliaga, Peregrina Eloína, Vinué Visús, David, \& Universitat Politècnica de València. Departamento de Ingeniería Cartográfica Geodesia y Fotogrametría - Departament d'Enginyeria Cartogràfica, Geodèsia i Fotogrametria. (n.d.). Análisis de la biomasa forestal mediante imágenes de satélite. Teledetección aplicada a la gestión forestal.

Parresol, B.R. (1999). "Assessing tree and stand biomass: A review with examples and critical comparisons”. Forest Science 45(4), 573-593.

Ritson, Peter \& Sochacki, Stanley. (2003). Measurement and prediction of biomass and carbon content of Pinus pinaster trees in farm forestry plantations, south-western Australia. Forest Ecology and Management. 175. 103-117. 10.1016/S0378-1127(02)00121-4.

Ruiz-Peinado, R., Río, M. del, Montero, G. (2011). "New models for estimating the carbon sink capacity of Spanish softwood species”. Forest Systems 20, 176-188.

Schlesinger, W .H. (1991). Biogeochemistry, an Analysis of Global Change. New York, USA, Academic Press. 